\title{
Unravelling the regulation pathway of photosynthetic AB-GAPDH
}

\author{
Dedicated to Emeritus Prof. Alberto Ripamonti
}

Roberto Marottaa,1, Alessandra Del Giudiceb,1, Libero Gurrieric, Silvia Fantid, Paolo Swuece, Luciano Galantinib, Giuseppe Falinid, Paolo Trost ${ }^{c}$, Simona Fermanid, ${ }^{,{ }^{*}},{ }^{*}$ Francesca Sparla ${ }^{\mathrm{c},{ }^{*}}$

${ }^{a}$ Electron Microscopy Facility (EMF), Italian Institute of Technology (IIT), 16163 Genova, Italy; bDepartment of Chemistry, University of Rome Sapienza, 00185 Rome, Italy; cDepartment of Pharmacy and Biotechnology-FaBiT, University of Bologna, 40126 Bologna, Italy; dDepartment of Chemistry G. Ciamician, University of Bologna, 40126 Bologna, Italy; eBiosciences Department, University of Milan, 20133 Milan, Italy; IInterdepartmental Centre for Industrial Research Health Sciences \& Technologies, University of Bologna, 40064 Bologna, Italy.

${ }^{1}$ These authors equally contributed to this work

*Simona Fermani and Francesca Sparla

E-mail: simona.fermani@unibo.it; francesca.sparla@unibo.it.

Author contributions: F.S., S.F. and P.T. designed research; R.M., A.D.G., L.G., S.F., L.G., S.F., and F.S. performed research; R.M., A.D.G., P.S., L.G., S.F., and F.S. analysed data; and R.M., A.D.G, G.F., L.G, P.T., S.F. and F.S. wrote the paper, all authors discussed the data and reviewed the manuscript.

Competing Interest Statement: The authors declare no competing interest.

Classification: Biological Science and Biochemistry.

Keywords: Photosynthesis; Calvin-Benson cycle; Redox regulation; Glyceraldehyde-3-phosphate dehydrogenase; Cryo-electron microscopy; Small angle X-ray scattering.

\section{This PDF file includes:}

Main Text

Figures 1 to 4 


\begin{abstract}
Oxygenic phototrophs perform carbon fixation through the Calvin-Benson cycle. Different mechanisms adjust the cycle and the light-harvesting reactions to rapid environmental changes. Photosynthetic glyceraldehyde 3-phosphate dehydrogenase (GAPDH) is a key enzyme of the cycle. In land plants, different photosynthetic GAPDHs exist: the most abundant formed by hetero-tetramers of $A$ and B-subunits, and the homo-tetramer $\mathrm{A}_{4}$. Regardless of the subunit composition, GAPDH is the major consumer of photosynthetic $\mathrm{NADPH}$ and for this reason is strictly regulated. While $\mathrm{A}_{4}-\mathrm{GAPDH}$ is regulated by $\mathrm{CP} 12, \mathrm{AB}-\mathrm{GAPDH}$ is autonomously regulated through the $\mathrm{C}$-terminal extension (CTE) of $\mathrm{B}$-subunits. Reversible inactivation of $\mathrm{AB}$ GAPDH occurs via oxidation of a cysteine pair located in the CTE, and substitution of NADP(H) with NAD $(H)$ in the cofactor binding domain. These combined conditions lead to a change in the oligomerization state and enzyme inactivation. SEC-SAXS and single-particle cryoEM analysis disclosed the structural basis of this regulatory mechanism. Both approaches revealed that $\left(A_{2} B_{2}\right) n-G A P D H$ oligomers with $n=1,2,4$ and 5 coexist in a dynamic system. $B$-subunits mediate the contacts between adjacent $A_{2} B_{2}$ tetramers in $A_{4} B_{4}$ and $\mathrm{A}_{8} \mathrm{~B}_{8}$ oligomers. The CTE of each $\mathrm{B}$-subunit penetrates into the active site of a $\mathrm{B}$-subunit of the adjacent tetramer, while the CTE of this subunit moves in the opposite direction, effectively preventing the binding of the substrate 1,3-bisphosphoglycerate in the B-subunits. The whole mechanism is made possible, and eventually controlled, by pyridine nucleotides. In fact, $N A D(H)$ by removing NADP $(H)$ from A-subunits allows the entrance of the CTE in B-subunits active sites and hence inactive oligomer stabilization.
\end{abstract}

\title{
Significance Statement
}

In land plants, glyceraldehyde 3-phosphate dehydrogenase (GAPDH) unique sink of reducing power of the entire Calvin-Benson cycle, is finely regulated. Based on the redox state and substrates concentration, its heteromeric form $A B-G A P D H$ oscillates between a fully active heterotetramer $\left(A_{2} B_{2}\right)$ and inactive oligomers. Experimental evidence demonstrates that GAPDH inactivation depends on the formation of dimers, tetramers or pentamers of $\mathrm{A}_{2} \mathrm{~B}_{2}$-modules, linked together by $\mathrm{C}$-terminal extensions (CTE) of B-subunits that extrude from one modular tetramer and occupy two active sites of the adjacent one. This molecular mechanism along with the unexpected observed dynamism of the system, shed light on how the CalvinBenson cycle is modulated in function of the light environmental changes. 


\section{Introduction}

Oxygenic photosynthesis sustains all life on Earth reducing carbon dioxide into carbohydrates while photooxidizing water into oxygen. The photosynthetic electron transport chain, strictly dependent on light, provides energy (ATP) and reducing power (NADPH) for the metabolic phase of the process. By consuming ATP and $\mathrm{NADPH}$, carbohydrates are produced from $\mathrm{CO}_{2}$ by the Calvin-Benson cycle (1-3). Despite the historical distinction between the two phases of photosynthesis, the entire process occurs during the day through a complex and diversified regulatory system that harmonizes the rate of carbon fixation with the rate of conversion of light energy into chemical energy (4-6).

Thioredoxins (TRXs) represent one of the wake-up calls of the Calvin-Benson cycle at dawn. Through the TRX/ferredoxin system part of the reducing power originated by the photosystem I induces the activation of the cycle in a TRX dependent manner (7-9). In land plants, phosphoribulokinase (PRK) (10-12), fructose 1,6-bisphosphatase (FBPase) (13, 14), sedoheptulose-1,7-bisphosphatase (SBPase) (14) and the ABisoform of glyceraldehyde 3-phosphate dehydrogenase (GAPDH) are direct target of TRXs $(15,16)$.

GAPDH catalyzes the only reducing step of the Calvin-Benson cycle and is the major consumer of the photosynthetically produced NADPH. Two isoforms of photosynthetic GAPDH coexist in the chloroplast stroma of land plants: a homo-tetramer exclusively made of $A$ subunits, and a hetero-tetramer containing both $A$ and $B$-subunits $(3,17)$ that can form higher order oligomers $(18,19)$. The structure of $A_{4}-$ and $A_{2} B_{2}-$ GAPDH is similar and highly conserved among GAPDHs $(20,21)$. Although the regulation of both isoforms occurs by interaction with CP12 and PRK, AB-GAPDH shows an additional autonomous regulation $(3,22)$.

CP12 is a small conditionally disordered protein containing two pairs of conserved cysteines $(23,24)$. The Cterminal pair, with a midpoint redox potential $\left(E_{\mathrm{m}, 7.9)}\right)$ of $-352 \mathrm{mV}$, binds $\mathrm{GAPDH}$, while the less negative potential $\mathrm{N}$-terminal disulfide $\left(E_{\mathrm{m}, 7.9}=-326 \mathrm{mV}\right)$ recruits PRK into the complex $(3,25)$. Recently, the structure of A-GAPDH/CP12/PRK complex has been solved, enlightening the molecular mechanisms involved in complex formation and redox regulation $(12,26,27)$.

AB-GAPDH performs the CP12-independent regulation through the presence of a 30 amino acid tail specific of the B-subunit $(3,15,21,28)$. This C-terminal extension (CTE) is highly homologous to the C-terminal region of $\mathrm{CP} 12$ and it has been postulated that the $\mathrm{B}$-subunit results from the fusion between the $\mathrm{A}$-subunit and the C-terminal half of CP12 $(3,29-31)$. Like CP12, also the CTE contains two cysteines that can be possibly engaged in a disulfide bridge.

AB-GAPDH exhibits its own propensity to vary the oligomeric state from active heterotetramers to inactive hexadecamers $(18,28,32,33)$. The transition between the oligomeric states depends not only on the redox state of the CTE, but also on the type of cofactor $(\operatorname{NADP}(\mathrm{H})$ or $\operatorname{NAD}(\mathrm{H}))$ and on the substrate 1,3bisphosphoglycerate (BPGA) availability $(15,34)$.

With the aim of disclosing the molecular mechanism that drives the oligomerization of AB-GAPDH, here we report a multi-approach structural study of the AB-GAPDH system by small angle X-ray scattering coupled with size exclusion chromatography (SEC-SAXS) and single-particle cryoelectron microscopy (cryoEM). Both experimental approaches highlight an unexpected dynamism of AB-GAPDH. Moreover, cryoEM reveals that pairs of B-subunits belonging to adjacent tetramers, mutually exchange their CTEs. Protruding like hooks, CTEs dock and penetrate in the active site of the adjacent tetramer blocking the access of the substrate.

\section{Results and Discussion}

Fingerprinting multiple oligomeric states of AB-GAPDH with SEC-SAXS. SEC-SAXS data were collected on active (NADP+-bound) and inactive (NAD+-bound) AB-GAPDH oligomers previously analyzed by Dynamic Light Scattering (DLS). Average hydrodynamic radius $\left(R_{h}\right)$ values of 52 and $100 \AA$ corresponding to apparent molecular weight (MW) of 159 and $736 \mathrm{kDa}$, were obtained for active and inactive forms, respectively. As a reference, the theoretical MW of $\mathrm{A}_{2} \mathrm{~B}_{2}-\mathrm{GAPDH}$ tetramers is $149 \mathrm{kDa}$. SAXS experiments showed that all samples presented a systematic variation of dimensional parameters, underlying the presence of different oligomers in addition to the more abundant $A_{2} B_{2}$ and $A_{8} B_{8}$ species expected in active and inactive samples, respectively (Fig. $1 A$ and $S /$ Appendix, Fig. S1) (15, 17, 21, 28). Statistically superimposable frames showing a constant gyration radius $\left(R_{g}\right)$ were identified and averaged to obtain representative SAXS profiles (Fig. $1 A$ and SI Appendix, Table S1) interpretable as AB-GAPDH oligomers on 
the basis of their dimensional parameters and distance distribution functions (P(r)) (Fig. 1 and $S I$ Appendix, Fig. S1 and Table S2).

In the inactive sample, the predominant species showed a $R_{g}$ of $67 \AA$, a maximum size ( $D_{\max }$ ) of $200 \AA$ and a MW between 500 and $600 \mathrm{kDa}$, compatible with the expected $\mathrm{A}_{8} \mathrm{~B}_{8}$ oligomer (SI Appendix, Table S1). In addition, a larger construct was identified, with a $R_{g}$ around $80 \AA$, a $D_{\max }$ of $280 \AA$ and an estimated MW between 650 and $700 \mathrm{kDa}$, suggesting an $\mathrm{A}_{10} \mathrm{~B}_{10}$ stoichiometry. A less abundant and smaller component was also observed at larger elution volumes (Fig. $1 A$, pink symbols). The estimation of its $\mathrm{R}_{\mathrm{g}}$ and $\mathrm{MW}$ was more uncertain. The related $P(r)$ profile showed a $D_{\max }$ around $150 \AA$ and only one maximum around $50 \AA$, clearly distinguishable from the bimodal $\mathrm{P}(\mathrm{r})$ function of $\mathrm{A}_{8} \mathrm{~B}_{8}$ (Fig. 1B). A similar $\mathrm{P}(\mathrm{r})$ profile (Fig. $1 C$ ) was calculated also at the beginning of the elution of the active-short sample again showing a wide range of estimated MWs (Fig. $1 A$ inset, purple diamonds). This sample was obtained from 2 hours incubation of the inactive sample under activating conditions (see SI Appendix, Materials and Methods). On this basis, the co-existence of species with intermediate stoichiometries possibly centered on $\mathrm{A}_{4} \mathrm{~B}_{4}$ and being in exchange equilibria with higher oligomers (inactive sample) or smaller oligomer (active-short sample) can be envisioned. The presence in vivo of the $\mathrm{A}_{4} \mathrm{~B}_{4}$ was already reported in different plant species $(33,35,36)$ besides the common $\mathrm{A}_{2} \mathrm{~B}_{2}$ and $\mathrm{A}_{8} \mathrm{~B}_{8}-\mathrm{GAPDH}$ forms, supporting the idea that this oligomer is not only an intermediate in the aggregation of $A_{2} B_{2}$ to $A_{8} B_{8}$, but even an essential player for $A B-G A P D H$ regulation.

The $\mathrm{R}_{\mathrm{g}}$ and $\mathrm{D}_{\max }$ (Fig. 1A, red symbols, SI Appendix, Fig. S1 and Table S2) detected at the elution maximum of the active-short sample $(14.8 \mathrm{ml})$ would suggest a fast-exchange dynamic equilibrium between $\mathrm{A}_{2} \mathrm{~B}_{2}$ and higher order oligomers (possibly involving $\mathrm{A}_{4} \mathrm{~B}_{4}$ as an intermediate species) in the elution conditions, giving rise to the impossibility to detect distinct SEC peaks (37).

The dimensional parameters of the active-short sample decreased gradually towards larger retention volumes and at the end of the elution, the structural parameters agreed with those found at the elution maximum of the active sample, i.e. a $R_{g}$ of $34 \AA$ and a $D_{\max }$ around $100 \AA$, compatible with an $A_{2} B_{2}$ tetramer (Fig. 1D).

Single-particle cryoEM analysis confirms the heterogeneity of inactive AB-GAPDH. In agreement with SAXS results, in inactivating conditions the single-particle analysis revealed the coexistence of different oligomeric states of the enzyme (Fig. 2). Projections related to different GAPDH oligomers, namely $A_{2} B_{2}$, $A_{4} B_{4}, A_{8} B_{8}$ and $A_{10} B_{10}$, were clearly present in negative stain and cryoEM micrographs (SI Appendix, Fig. S2). They were also present in the $2 \mathrm{D}$ and $3 \mathrm{D}$ classifications performed on the complete GAPDH data set (Figs. 2A and SI Appendix, Fig. S3A).

An estimation of the relative abundance of each oligomer obtained from the number of refined particles, showed that the $\mathrm{A}_{8} \mathrm{~B}_{8}$ hexadecamer is the most abundant species (42\%), albeit in two distinct conformers, named main (29\%) and alternative (13\%) (Fig. 2B). The $A_{4} B_{4}$ octamer (25\%) and the $A_{2} B_{2}$ tetramer (24\%) are less abundant. The remaining $9 \%$ corresponds to the $\mathrm{A}_{10} \mathrm{~B}_{10}$ icosamer.

The cryoEM density map of the $\mathrm{A}_{2} \mathrm{~B}_{2}$ tetramer was determined at $6.3 \AA$ (Fig. $2 B$ and SI Appendix, Fig.S4). Its superimposition to the crystal structure of oxidized $A_{2} B_{2}-G A P D H$ (PDB ID 2PKQ) (21) does not point out significant conformational differences.

The $8.9 \AA \mathrm{A}_{4} \mathrm{~B}_{4}$ cryoEM density map is a dimer with c1 symmetry formed by two $\mathrm{A}_{2} \mathrm{~B}_{2}$ tetramers rotated each other by approximately $180^{\circ}$ (Figs. $2 B$ and $3 A$ and $S I$ Appendix Figs. S5A, B). Imposition of c2 symmetry in the $3 \mathrm{D}$ refinement process produced a less resolved reconstruction.

The $\mathrm{A}_{8} \mathrm{~B}_{8}$ hexadecamer exists in two conformations, both with $\mathrm{c} 2$ symmetry and formed by two $\mathrm{A}_{4} \mathrm{~B}_{4}$ dimers. The $5.7 \AA$ cryoEM density map of the main conformer shows a central cavity with an area of about $1763 \AA^{2}$ (Figs. $2 B$ and $3 D-I$ and SI Appendix Fig. S5C). Compared to the main conformer, the two $\mathrm{A}_{4} \mathrm{~B}_{4}$ dimers of the alternative conformer (solved at $7.1 \AA$ ) are slightly shifted in the $\mathrm{x}$ direction, one in respect to the other, and the central cavity has a similar area (1738 $\left.\AA^{2}\right)$ (Fig. 2B and SI Appendix, Fig. S6A).

Finally, the $13 \AA A_{10} B_{10}$ electron density map is a pentamer with c5 symmetry and a central $5531 \AA^{2}$ wide seastar-shaped cavity (Fig. 2B and SI Appendix, Fig. S7A). 
bioRxiv preprint doi: https://doi.org/10.1101/2021.11.21.469434; this version posted November 21, 2021. The copyright holder for this preprint (which was not certified by peer review) is the author/funder. All rights reserved. No reuse allowed without permission.

In all oligomers, the contacts between $\mathrm{A}_{2} \mathrm{~B}_{2}$ tetramers are always mediated by $\mathrm{B}$-subunits as shown by rigidly fitting the oxidized $\mathrm{A}_{2} \mathrm{~B}_{2}$ crystal structure (PDB ID 2PKQ) (21) inside their respective cryoEM density maps (Figs. 2B, 3A, $B$ and $E-H$ and SI Appendix, Figs. S6A-C and S7A-C). Although A- and B-subunits show a high sequence identity (about 81\%; SI Appendix, Fig. S8) and similar overall structure, the positioning of Bsubunit rather than $\mathrm{A}$-subunit at the contact regions between adjacent tetramers, gave significantly higher correlation coefficients (SI Appendix, Table S3). Consistently, the capability of AB-GAPDH to aggregate is long known to depend on the CTE, which is exclusive of B-subunits $(15,34)$.

Dissecting the assembling of $A_{2} B_{2}-G A P D H$ tetramers in higher order oligomers: the role of the CTE. The cryoEM density maps of $\mathrm{A}_{4} \mathrm{~B}_{4}$ and both conformers of $\mathrm{A}_{8} \mathrm{~B}_{8}$ show unassigned densities in proximity of the contact regions between adjacent $\mathrm{A}_{2} \mathrm{~B}_{2}$ tetramers (Fig. 3 and SI Appendix, Fig. S6A-C). These densities start from the last residue of the B-subunit of the crystal structure and continue in the catalytic domain of the closest B-subunit of the adjacent tetramer about $20 \AA$ far away. In some cases the density was clearly visible and continuous, in others was less defined. A model of the $\mathrm{C}_{\alpha}$ backbone of the CTE, including the side chains of Cys349 and Cys358 forming the regulative disulfide bridge, was built on the basis of the electron density map of the $\mathrm{A}_{8} \mathrm{~B}_{8}-\mathrm{GAPDH}$ main conformer. The model consists of an extended linker region visible in the electron density maps at lower density threshold, followed by a helix, a circular motif determined by the disulfide bond and a final random coil region (Figs. 3B, $F, H$ and $4 A$ ). In all GAPDH oligomers the CTEs mediate the connection between $B$-subunits belonging to adjacent $A_{2} B_{2}$ tetramers, and each tetramer is connected with the adjacent one by two CTEs. The CTE belonging to one B-subunit penetrates into the catalytic domain of the B-subunit of the adjacent tetramer whose CTE in turn enters into the catalytic domain of the B-subunit of the first tetramer in the opposite direction (Figs. $3 A, B$ and $D$ - $H$ and $S I$ Appendix, Figs. $\mathrm{S} 6 B$ and $C$ ). The catalytic sites of the A-subunits, two per tetramer, remain free.

The CTE linker regions (Figs. 3B, F, H and SI Appendix, Fig. S6C) differ significantly from each other in length (from $15 \AA$ to $22 \AA$ ) and conformation among and inside the different oligomers. They are indeed highly flexible as corroborated by ResMap results that pointed to a significant decrease in resolution in the CTE linker regions (Fig. 3C, I and SI Appendix, Fig. S6D).

The $A_{2} B_{2}$ and $A_{4} B_{4}$ are able to form higher oligomers, having two "non-engaged" CTEs that are likely free to move in the surroundings and therefore not observed in their corresponding electron density maps (Fig. 3A and SI Appendix, Fig. S4A). Consistently, the chimeric form composed of A-subunits fused with CTE $[(A+C T E) 4]$ makes oligomers that reach an unexpectedly high molecular mass, at least 7 -fold bigger than the corresponding tetramer $(15,34)$.

Considering that the $\mathrm{A}_{8} \mathrm{~B}_{8}$ oligomer shows each available CTE engaged with another $\mathrm{B}$-subunit (Figs. 3D- $H$ and SI Appendix, Fig. S6A-C), it is suggested to be the end-point of the oligomerization process. A similar situation is probably present in the $A_{10} B_{10}$, but the limited resolution of the electron density map prevented the CTE reconstruction ( $S$ I Appendix, Fig. S7A-D).

The last portion of the CTE (helix, circular motif and terminal random coil) of each B-subunit penetrates into the catalytic site of a B-subunit of the adjacent tetramer through the large cavity formed between the bound cofactor $\operatorname{NAD}(\mathrm{H})$ and its S-loop (Fig. 4A), ending in the $P_{s}$ site that hosts the phosphate group of the substrate and very close to the hydroxyl groups of the nicotinamide ribose (Fig. 4B). Therefore, the CTE prevents the access and binding of the substrate in the B-subunit active site. Arginines 195 and 231 involved in the stabilization of the $P_{s}$ site (21), likely interact with the backbone and side chain carboxylate group of CTE C-terminal Glu362. Additional interactions are likely formed between the positively charged residues of the S-loop such as Arg183 and His190 and negative residues of the CTE (Asp355, Glu356 and Glu357) (Fig. $4 B$ and SI Appendix, Fig. S8). Moreover, Glu356 and Glu357 are located close to the ribose hydroxyl groups of the NAD adenine moiety of the adjacent A-subunit and their extended and negatively charged side chain can possibly interfere with the correct positioning of the NADP+ 2'-phosphate group. This explains why the enzyme needs to replace $\operatorname{NADP}(\mathrm{H})$ by $\operatorname{NAD}(\mathrm{H})$ in order to assemble in oligomers and why the phosphate cofactor helps the oligomers dissociation.

The cavity occupied by the CTE in $\mathrm{A}_{8} \mathrm{~B}_{8}$ cryoEM structure is the same observed in the crystal structure of oxidized $\mathrm{A}_{2} \mathrm{~B}_{2}$ complexed with $\mathrm{NADP}^{+}$(PDB ID 2PKQ) (21). In this last structure, it was possible to build only less than ten C-terminal residues of the two CTEs belonging to the B-subunits of the tetramer. Nevertheless, the superimposition of the two structures shows that the last portion of CTE has a different conformation and 
in oxidized $\mathrm{A}_{2} \mathrm{~B}_{2}-\mathrm{GAPDH}$ complexed with $\mathrm{NADP}^{+}$ends in the more external region of the catalytic cavity leaving free the $\mathrm{P}_{\mathrm{s}}$ and the $\mathrm{P}_{\mathrm{i}}$ sites (Fig. 4C) $(21,38)$.

The CTE responsible of all regulatory properties of $A_{2} B_{2}-G A P D H$, is considered evolutionary derived from CP12, being homologous to the C-terminal domain of $\mathrm{CP} 12(15,32)$. The structural models of the binary $\mathrm{A}_{4-}$ GAPDH/CP12 and ternary $\mathrm{A}_{4}-\mathrm{GAPDH} / \mathrm{CP} 12 / \mathrm{PRK}$ complexes $(12,27,39,40)$ reveal that the CTE in $\mathrm{A}_{8} \mathrm{~B}_{8}-$ GAPDH and the C-terminal domain of CP12 share not only the same cavity but also a very similar conformation (Fig. 4D). Indeed, the C-terminal domain of CP12 fits well inside the unassigned density of each B-subunit and particularly the $\alpha$-helix portion appears well superimposable. The unique striking difference is that CP12 penetrates more deeply in the GAPDH active site compared to CTE, blocking both $\mathrm{P}_{\mathrm{s}}$ and the $\mathrm{P}_{\mathrm{i}}$ sites. Indeed, the side chain of Asn78, the last CP12 residue, is observed at an $\mathrm{H}$-bond distance from the thiol group of the catalytic Cys149 $(12,39,40)$.

Interface analysis of $A B$-oligomers. The $A_{2} B_{2}$ tetramers within oligomers are linked together by the CTEs but appear to interact also through a different surface. PDBePISA (41) calculations show that in all GAPDH oligomers the CTEs contribute to the interface area between $A_{2} B_{2}$ tetramers by $39 \%$ in $A_{4} B_{4}, 32 \%$ and $33 \%$ in $\mathrm{A}_{8} \mathrm{~B}_{8}$ and its alternative conformer, respectively (SI Appendix, Table S4).

$\mathrm{A}_{8} \mathrm{~B}_{8}$ oligomer shows the largest total interface area $\left(2641 \AA^{2}\right)$ and consequently the largest average single interface area equal to $660 \AA^{2}$ (449 $\AA^{2}$ without CTE). This area decreases to $625 \AA^{2}\left(421 \AA^{2}\right.$ without CTE) in the case of the alternative conformer and to $656 \AA^{2}\left(403 \AA^{2}\right.$ without CTE) for $A_{4} B_{4}$. The $A_{10} B_{10}$ has the smallest average single interface area $\left(228 \AA^{2}\right)$.

CTE-independent interacting surfaces are similar in all oligomers and invariably include four stretches of residues (77-80; 97-114; 119-127; 139-143) located in $\alpha$-helices and loops (Fig. 3, SI Appendix Figs. S6C and S8). The last two stretches contain two amino acid insertions in B- compared to A-subunit (Ser123A and Val140) and various sequence differences (SI Appendix Fig. S8). This may explain (42) why artificial tetramers made of $B$-subunits only $\left(B_{4}\right)$ or $(A+C T E)_{4}$ form oligomers of different size under inactivating conditions (491 vs >1800 kDa, respectively) $(15,32,34)$.

In $A_{4} B_{4}$ and $A_{8} B_{8}$ oligomers, but not in $A_{10} B_{10}$, additional interface regions comprise residues from the $S$-loop (179-195) and residues between strands $\beta_{2}$ and $\beta_{3}$ (206-208 and 215-222).

Intriguingly, the CTEs also play a key role in improving the thermodynamic stability of both $A_{4} B_{4}$ and $A_{8} B_{8}$ oligomers. The calculated dissociation free energy $\left(\Delta \mathrm{G}_{\text {diss }}\right)$ is negative in all oligomers without $\mathrm{CTES}$ indicating that they are unstable, while the presence of CTE prevents their dissociation (SI Appendix, Table S4). The most stable oligomer is $A_{8} B_{8}$ in the main conformation $\left(\Delta G_{\text {diss }}=41 \mathrm{kcal} / \mathrm{mol}\right.$ ), followed by $A_{4} B_{4}$ $\left(\Delta G_{\text {diss }}=35.9 \mathrm{kcal} / \mathrm{mol}\right)$ and the hexadecamer alternative conformer $\left(\Delta \mathrm{G}_{\text {diss }}=35.5 \mathrm{kcal} / \mathrm{mol}\right)$.

SEC-SAXS data matching with AB-structural models. The theoretical scattering profiles of cryoEM models of the AB-GAPDH oligomers (here presented), and the $A_{2} B_{2}$ crystal structure (PDB ID 2PKQ) (21) were calculated (SI Appendix, Fig. S9) to evaluate the agreement with SEC-SAXS data and the contribution of the different oligomers.

The inactive sample relative abundance (particles percentage of $19 \%, 49 \%, 30 \%$ and $2 \%$ for $A_{10} B_{10}, A_{8} B_{8}$, $A_{4} B_{4}$. and $A_{2} B_{2}$,respectively) shows a general agreement with the cryoEM data, except for the negligible contribution of $\mathrm{A}_{2} \mathrm{~B}_{2}$ and a larger fraction of $\mathrm{A}_{10} \mathrm{~B}_{10}$ (Fig. $2 B$; SI Appendix, Fig. S10A, D). The comparison between the theoretical and experimental scattering profiles suggests that the data from the inactive sample can be also interpreted reasonably well in terms of one prevailing oligomer at their elution maxima i.e. $\mathrm{A}_{10} \mathrm{~B}_{10}, \mathrm{~A}_{8} \mathrm{~B}_{8}$ and $\mathrm{A}_{4} \mathrm{~B}_{4}$ (SI Appendix, Fig. S10E; grey vs. black line). The $\mathrm{A}_{4} \mathrm{~B}_{4}$ coexists with the predominant $\mathrm{A}_{8} \mathrm{~B}_{8}$ in a rapid exchanging process and its scattering became dominant only at the tail of the elution $(S /$ Appendix, Fig. S10A).

Data from the active sample are well interpreted by the scattering profile of the $\mathrm{A}_{2} \mathrm{~B}_{2}$ tetramer (SI Appendix, Fig. S10C, $D$ and $G$ and Table S5), while the active-short sample consists of a more complex mixture, predominantly composed by the $A_{2} B_{2}$ form coexisting with a significant fraction of $A_{4} B_{4}$ oligomer and $A B$ dimers (SI Appendix, Fig. S10B, F). The introduction of this last form already described for non- 
photosynthetic GAPDHs (43, 44), clearly improved the fitting (SI Appendix, Fig. S10F; black vs. grey line). However, the absence in the experimental data of the pronounced minimum observed at $q=0.1 \AA^{-1}$ in the $\mathrm{A}_{2} \mathrm{~B}_{2}$ theoretical scattering profile, can also be ascribed to a quaternary structure rearrangement in solution, that generates a less compact and isometric tetramer $(26,45)$.

Concentration effect on the oligomerization of AB-GAPDH. SAXS measurements without SEC separation (SC-SAXS) on AB-GAPDH in inactive and active conditions were also performed (SI Appendix, Table S6). The inactive sample can be described as a mixture in which the $\mathrm{A}_{10} \mathrm{~B}_{10}$ oligomer is predominant (roughly $50 \%$ volume fraction), coexisting with the $\mathrm{A}_{8} \mathrm{~B}_{8}$ oligomer (35\%) and a smaller fraction of the $\mathrm{A}_{4} \mathrm{~B}_{4}$ form (15\%) (SI Appendix, Fig. S11A, B and C).

In the active sample, a systematic decrease of the average dimensions and forward scattered intensity was observed with the decrease of the protein concentration (SI Appendix, Table S6). The P( $r$ ) functions underwent a systematic decrease of the additional peak at $100 \AA$ seen in the bimodal $P(r)$ of higher oligomers, in favor of the main peak at $50 \AA$ characteristic of the $\mathrm{A}_{2} \mathrm{~B}_{2}$ tetramer (SI Appendix, Fig. S11D). The data fitting in terms of a mixture suggests that the fraction of $\mathrm{A}_{2} \mathrm{~B}_{2}$ increased from roughly $20 \%$ to above $60 \%$ upon dilution, at the expenses of the $A_{4} B_{4}$ and $A_{10} B_{10}$ oligomers, present as $50 \%$ and $27 \%$ volume fractions, respectively, in the most concentrated sample (SI Appendix, Fig. S11E, F and Table S7).

This analysis shows that the cryoEM models explain a consistent amount of the SAXS signal. However, the AB-GAPDH oligomerization equilibrium in solution appears more complex. Indeed, partially formed oligomers or less symmetric conformations of $\left(A_{2} B_{2}\right)_{n}(n=4$ and 5$)$ oligomers such as polymeric chains of $A_{2} B_{2}$ units with free CTEs, and small fractions of larger assemblies $(n>5)$, could explain the non-optimal agreement of the fits based on the cryoEM models only and the maximum sizes larger than $240 \AA$ (expected for the $A_{10} B_{10}$ oligomer) detected in the inactive sample.

\section{Concluding remarks}

NAD $(\mathrm{P}) \mathrm{H}$-dependent GAPDH enzymes are involved in photosynthetic carbon assimilation of all oxygenic phototrophs. However, whereas cyanobacteria and most eukaryotic algae exclusively present a homotetrameric form $\left(A_{4}-G A P D H\right)$, the major chloroplast GAPDH isozyme of land plants is formed by $A$ and $B$ subunits, the latter containing a redox-sensitive C-terminal extension (CTE) which controls the NADPHdependent activity of the enzyme and the capability to form higher order oligomers $(15,32)$.

In this study, we have structurally characterized photosynthetic AB-GAPDH and disclosed the CTE-mediated regulation/oligomerization process, by combining SEC-SAXS and single-particle cryoEM analysis. Both experimental approaches highlighted the presence in both active and inactive in vitro conditions (mimicking light and dark in vivo conditions) of various oligomers in addition to the expected species with $\mathrm{A}_{2} \mathrm{~B}_{2}$ and $\mathrm{A}_{8} \mathrm{~B}_{8}$ stoichiometries, respectively $(15,17,21,28)$. In activating conditions beside the heterotetramer $A_{2} B_{2}$, the octamer $\mathrm{A}_{4} \mathrm{~B}_{4}$ was detected, while in inactivating conditions the population increases to four species, i.e. $\left(A_{2} B_{2}\right)_{n}$ with $n=1,2,4$ and 5 (Figs. 1 and 2). The unexpected dynamism of the AB-GAPDH system is not simply ascribable to the experimental conditions. Indeed, $A_{4} B_{4}$ oligomers were observed in leaves of different plant species $(35,46)$, indicating that this form is both an intermediate step in GAPDH oligomerization and an essential player in its regulation. Moreover, being $A_{4} B_{4}$ a structural unit of $A_{8} B_{8}$ and likely of $A_{10} B_{10}$ oligomers, it represents for the $A B-G A P D H$ system a ubiquitous reservoir of inactive $A_{2} B_{2}$ tetramers that when needed can easily dissociate to form the active species or aggregate in higher molecular weight oligomers.

In all oligomers, the interfaces between $A_{2} B_{2}$-tetramers uniquely involve $B$-subunits (Figs. $2 B$ and 3 ), confirming that the CTE manages the AB-GAPDH assembly process upon $N A D P(H) / N A D(H)$ cofactor exchange. Moreover, the higher resolution $\mathrm{A}_{4} \mathrm{~B}_{4}$ and $\mathrm{A}_{8} \mathrm{~B}_{8}$ cryoEM models show that pair of $\mathrm{B}$-subunits from adjacent tetramers hug each other through their CTEs (Figs 3A, B, E-H, SI Appendix Fig. S6B, C). Each CTE slips into the cofactor cavity of the partner B-subunit up to its catalytic $P_{s}$ site, effectively preventing the substrate binding (Fig. 4). This positioning of the CTE is only possible if $N A D(H)$ is bound to the A-subunit. However, $\operatorname{NAD}(\mathrm{H})$ does not promote oligomerization directly, but it does so by replacing $\operatorname{NADP}(\mathrm{H})$. Indeed, the 2'-phosphate of $\operatorname{NADP}(\mathrm{H})$ is apparently incompatible with the allocation of the CTE in the active site of B- 
subunits, justifying the disassembling role of this cofactor (15). On the other hand, the catalytic sites of Asubunits are free and likely available to perform the constitutive $\mathrm{NADH}$-dependent catalysis.

The conformation assumed by the last portion of the CTE closely resembles that one of the CP12 C-terminal domain in the GAPDH-CP12-PRK ternary complex (PDB ID 6GVE) (27) (Fig. 4C), indicating that the molecular strategy for the modulation of GAPDH activity appears conserved among all photosynthetic GAPDHs.

In conclusion, our structural study provides a full picture at molecular level showing how the dynamic changes in the oligomeric status of AB-GAPDH contribute to the modulation of the Calvin-Benson cycle in response to light conditions occurring in the natural environment.

\section{Materials and Methods}

Preparation of AB-GAPDH oligomers. AB-GAPDH isoforms were prepared from partially purified spinach chloroplasts, following ammonium sulfate precipitation, cold acetone precipitation and anion exchange chromatography, as described in (21). Active (NADP+-bound) and inactive (NAD+-bound) AB-GAPDH samples were obtained as reported in SI Appendix, Materials and Methods.

Small Angle X-ray Scattering data collection and analysis. SAXS data were collected at the BioSAXS beamline BM29 at the European Synchrotron Radiation Facility (ESRF), Grenoble (France) (47). Details of the samples composition, experimental procedures and data acquisition parameters and analysis are reported in SI Appendix, Materials and Methods.

Theoretical scattering profiles calculation from 3D Data. Coordinates from the crystal structure of $A_{2} B_{2-}$ GAPDH (PDB ID 2PKQ) (21) and from the cryoEM models of AB-GAPDH oligomers (present work) were used to calculate theoretical scattering profiles as detailed in SI Appendix, Materials and Methods.

CryoEM data collection and analysis. Purified inactive AB-GAPDH sample was adsorbed onto a Quantifoil holey TEM grids and plunge-frozen in liquid ethane with a Vitrobot Mark IV cryo-plunger. CryoEM data were collected on a Tecnai F30 Polara cryo electron microscope at $300 \mathrm{keV}$. AB-GAPDH oligomers were reconstructed in RELION $3.0(48,49)$ and refined in the real-space. Experimental procedures, data analysis and modeling are reported in SI Appendix, Materials and Methods.

Data Availability. The cryoEM maps of AB-GAPDH oligomers and the coordinates of atomic models generated and analyzed in the current study, have been deposited in the Electron Microscopy Data Bank and in the Protein Data Bank, under accession codes: EMD-13824 and PDB ID 7Q53 for $\mathrm{A}_{2} \mathrm{~B}_{2}$; EMD-13825 and PDB ID 7Q54 for $\mathrm{A}_{4} \mathrm{~B}_{4}$; EMD-13826 and PDB ID 7Q55 for $\mathrm{A}_{8} \mathrm{~B}_{8}$ (main conformer); EMD-13827 and PDB ID 7Q56 for $\mathrm{A}_{8} \mathrm{~B}_{8}$ (alternative conformer), EMD-13828 and PDB ID 7Q57 for $\mathrm{A}_{10} \mathrm{~B}_{10}$.

\section{Acknowledgements}

We deeply thank Prof. Viorel Nicolae Pavel for his essential suggestions on SAXS experiments and data analysis. This work has been supported by Instruct, project number PID 1829 "Unravelling the pathway of regulation of photosynthetic AB-GAPDH by cryo-EM" funded by the Horizon 2020 programme of the European Union. The high-resolution data were collected at the IBS - Institut de Biologie Structurale in Grenoble (France) with assistance from Dr. Guy Schoehn. We thank the European Synchrotron Radiation Facility for allocation of SAXS beam time (BAG Proposals MX1750) and the staff of beamline BM29 for technical support. S.F. and G.F. thanks the Consorzio Interuniversitario di Ricerca in Chimica dei Metalli nei Sistemi Biologici (CIRCMSB). 


\section{References}

1. L. Michelet, et al., Redox regulation of the Calvin-Benson cycle: Something old, something new. Frontiers in Plant Science 4 (2013).

2. M. P. Johnson, Photosynthesis. Essays in Biochemistry 60, 255-273 (2016).

3. L. Gurrieri, S. Fermani, M. Zaffagnini, F. Sparla, P. Trost, Calvin-Benson cycle regulation is getting complex. Trends in Plant Science (2021) https:/doi.org/10.1016/j.tplants.2021.03.008.

4. R. Scheibe, K.-J. Dietz, Reduction-oxidation network for flexible adjustment of cellular metabolism in photoautotrophic cells. Plant, Cell \& Environment 35 (2012).

5. J. Minagawa, R. Tokutsu, Dynamic regulation of photosynthesis in Chlamydomonas reinhardtii. The Plant Journal 82 (2015).

6. E. Heyneke, A. R. Fernie, Metabolic regulation of photosynthesis. Biochemical Society Transactions 46 (2018).

7. B. B. Buchanan, Regulation of $\mathrm{CO} 2$ assimilation in oxygenic photosynthesis: The ferredoxin/thioredoxin system. Archives of Biochemistry and Biophysics 288 (1991).

8. H. C. Huppe, F. de Lamotte-Guéry, J.-P. Jacquot, B. B. Buchanan, The ferredoxin-thioredoxin system of a green alga, Chlamydomonas reinhardtii. Planta 180 (1990).

9. L. Nikkanen, E. Rintamäki, Chloroplast thioredoxin systems dynamically regulate photosynthesis in plants. Biochemical Journal 476 (2019).

10. H. K. Brandes, F. W. Larimer, F. C. Hartman, The Molecular Pathway for the Regulation of Phosphoribulokinase by Thioredoxin f. Journal of Biological Chemistry 271 (1996).

11. L. Gurrieri, et al., Arabidopsis and Chlamydomonas phosphoribulokinase crystal structures complete the redox structural proteome of the Calvin-Benson cycle. Proceedings of the National Academy of Sciences of the United States of America 116 (2019).

12. A. Yu, et al., Photosynthetic phosphoribulokinase structures: Enzymatic mechanisms and the redox regulation of the Calvin-Benson-Bassham cycle. Plant Cell 32, 1556-1573 (2020).

13. M. Chiadmi, Redox signalling in the chloroplast: structure of oxidized pea fructose-1,6-bisphosphate phosphatase. The EMBO Journal 18 (1999).

14. D. D. Gütle, et al., Chloroplast FBPase and SBPase are thioredoxin-linked enzymes with similar architecture but different evolutionary histories. Proceedings of the National Academy of Sciences of the United States of America 113, 6779-6784 (2016).

15. F. Sparla, P. Pupillo, P. Trost, The C-terminal extension of glyceraldehyde-3-phosphate dehydrogenase subunit $B$ acts as an autoinhibitory domain regulated by thioredoxins and nicotinamide adenine dinucleotide. Journal of Biological Chemistry 277, 44946-44952 (2002).

16. R. A. Wolosiuk, B. B. Buchanan, Regulation of chloroplast phosphoribulokinase by the ferredoxin/thioredoxin system. Archives of Biochemistry and Biophysics 189 (1978). 
17. S. Scagliarini, The non-regulatory isoform of NAD(P)-glyceraldehyde-3-phosphate dehydrogenase from spinach chloroplasts. Journal of Experimental Botany 49 (1998).

18. P. PUPILLO, G. G. PICCARI, The Reversible Depolymerization of Spinach Chloroplast GlyceraldehydePhosphate Dehydrogenase. Interaction with Nucleotides and Dithiothreitol. European Journal of Biochemistry 51 (1975).

19. B. B. BUCHANAN, R. A. WOLOSIUK, Photosynthetic regulatory protein found in animal and bacterial cells. Nature 264 (1976).

20. S. Fermani, et al., Crystal structure of the non-regulatory A4-isoform of spinach chloroplast glyceraldehyde-3-phosphate dehydrogenase complexed with NADP. Journal of Molecular Biology 314 (2001).

21. S. Fermani, et al., Molecular mechanism of thioredoxin regulation in photosynthetic A2B2glyceraldehyde-3-phosphate dehydrogenase. Proceedings of the National Academy of Sciences of the United States of America 104 (2007).

22. A. E. Carmo-Silva, M. E. Salvucci, The activity of Rubisco's molecular chaperone, Rubisco activase, in leaf extracts. Photosynthesis Research 108 (2011).

23. D. Reichmann, U. Jakob, The roles of conditional disorder in redox proteins. Current Opinion in Structural Biology 23 (2013).

24. H. Launay, et al., Cryptic Disorder Out of Disorder: Encounter between Conditionally Disordered CP12 and Glyceraldehyde-3-Phosphate Dehydrogenase. Journal of Molecular Biology 430 (2018).

25. L. Marri, et al., In vitro characterization of Arabidopsis CP12 isoforms reveals common biochemical and molecular properties. Journal of Plant Physiology 167 (2010).

26. A. del Giudice, et al., Unravelling the shape and structural assembly of the photosynthetic GAPDHCP12-PRK complex from Arabidopsis thaliana by small-angle X-ray scattering analysis. Acta Crystallographica Section D: Biological Crystallography 71 (2015).

27. C. R. McFarlane, et al., Structural basis of light-induced redox regulation in the Calvin-Benson cycle in cyanobacteria. Proceedings of the National Academy of Sciences of the United States of America 116, 20984-20990 (2019).

28. R. Scheibe, E. Baalmann, J. E. Backhausen, C. Rak, S. Vetter, C-terminal truncation of spinach chloroplast NAD(P)-dependent glyceraldehyde-3-phosphate dehydrogenase prevents inactivation and reaggregation. Biochimica et Biophysica Acta (BBA) - Protein Structure and Molecular Enzymology 1296 (1996).

29. P. Trost, et al., Thioredoxin-dependent regulation of photosynthetic glyceraldehyde-3-phosphate dehydrogenase: autonomous vs. CP12-dependent mechanisms. Photosynthesis Research 89 (2006).

30. N. Wedel, J. Soll, Evolutionary conserved light regulation of Calvin cycle activity by NADPH-mediated reversible phosphoribulokinase/CP12/glyceraldehyde-3-phosphate dehydrogenase complex dissociation. Proceedings of the National Academy of Sciences 95 (1998). 
31. J. Petersen, R. Teich, B. Becker, R. Cerff, H. Brinkmann, The GapA/B Gene Duplication Marks the Origin of Streptophyta (Charophytes and Land Plants). Molecular Biology and Evolution 23 (2006).

32. E. Baalmann, R. Scheibe, R. Cerff, W. Martin, Functional studies of chloroplast glyceraldehyde-3phosphate dehydrogenase subunits $A$ and $B$ expressed in Escherichia coli: formation of highly active A4 and B4 homotetramers and evidence that aggregation of the B4 complex is mediated by the B subunit carboxy terminus. Plant Molecular Biology 32 (1996).

33. T. P. Howard, M. Metodiev, J. C. Lloyd, C. A. Raines, Thioredoxin-mediated reversible dissociation of a stromal multiprotein complex in response to changes in light availability. Proceedings of the National Academy of Sciences 105 (2008).

34. F. Sparla, et al., Regulation of photosynthetic GAPDH dissected by mutants. Plant Physiology 138, 2210-2219 (2005).

35. T. P. Howard, J. C. Lloyd, C. A. Raines, Inter-species variation in the oligomeric states of the higher plant Calvin cycle enzymes glyceraldehyde-3-phosphate dehydrogenase and phosphoribulokinase. Journal of Experimental Botany 62, 3799-3805 (2011).

36. E. Baalmann, J. E. Backhausen, C. Kitzmann, R. Scheibe, Regulation of NADP-Dependent Glyceraldehyde 3-Phosphate Dehydrogenase Activity in Spinach Chloroplasts*. Botanica Acta 107 (1994).

37. F. J. Stevens, Analysis of protein-protein interaction by simulation of small-zone size exclusion chromatography. Stochastic formulation of kinetic rate contributions to observed high-performance liquid chromatography elution characteristics. Biophysical Journal 55 (1989).

38. F. Sparla, et al., Coenzyme site-directed mutants of photosynthetic $A<\inf >4</$ inf $>-G A P D H$ show selectively reduced NADPH-dependent catalysis, similar to regulatory AB-GAPDH inhibited by oxidized thioredoxin. Journal of Molecular Biology 340 (2004).

39. H. Matsumura, et al., Structure basis for the regulation of glyceraldehyde-3-phosphate dehydrogenase activity via the intrinsically disordered protein CP12. Structure 19, 1846-1854 (2011).

40. S. Fermani, et al., Conformational selection and folding-upon-binding of intrinsically disordered protein CP12 regulate photosynthetic enzymes assembly. Journal of Biological Chemistry 287 (2012).

41. E. Krissinel, K. Henrick, Inference of Macromolecular Assemblies from Crystalline State. Journal of Molecular Biology 372 (2007).

42. K. Hashimoto, A. R. Panchenko, Mechanisms of protein oligomerization, the critical role of insertions and deletions in maintaining different oligomeric states. Proceedings of the National Academy of Sciences 107 (2010).

43. O. Roitel, P. Vachette, S. Azza, G. Branlant, P but not R-axis Interface is Involved in Cooperative Binding of NAD on Tetrameric Phosphorylating Glyceraldehyde-3-phosphate Dehydrogenase from Bacillus stearothermophilus. Journal of Molecular Biology 326 (2003). 
44. C. M. Torres-Bugeau, et al., Characterization of Heparin-induced Glyceraldehyde-3-phosphate Dehydrogenase Early Amyloid-like Oligomers and Their Implication in $\alpha$-Synuclein Aggregation*. Journal of Biological Chemistry 287 (2012).

45. F. Ferreira-da-Silva, et al., The Crystal and Solution Structures of Glyceraldehyde-3-phosphate Dehydrogenase Reveal Different Quaternary Structures. Journal of Biological Chemistry 281 (2006).

46. T. P. Howard, M. Metodiev, J. C. Lloyd, C. A. Raines, Thioredoxin-mediated reversible dissociation of a stromal multiprotein complex in response to changes in light availability. Proceedings of the National Academy of Sciences 105 (2008).

47. P. Pernot, et al., Upgraded ESRF BM29 beamline for SAXS on macromolecules in solution. Journal of Synchrotron Radiation 20 (2013).

48. S. H. W. Scheres, Processing of Structurally Heterogeneous Cryo-EM Data in RELION. Methods in enzymology 579 (2016).

49. J. Zivanov, et al., New tools for automated high-resolution cryo-EM structure determination in RELION-3. elife 7 (2018). 


\section{Figures}
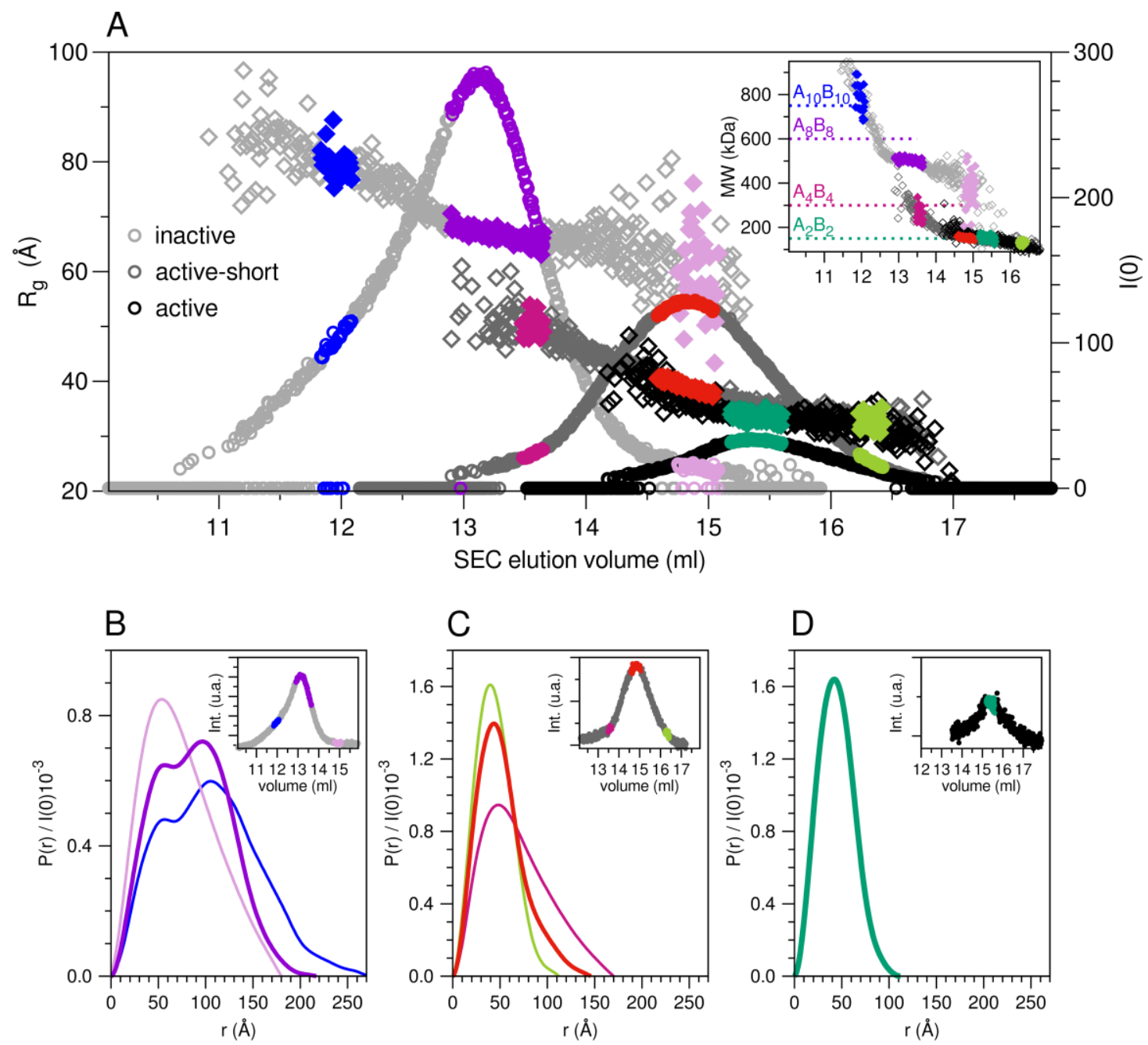

Figure 1. SEC-SAXS elution profiles and $P(r)$ functions of AB-GAPDH species.

(A) Parameters derived from the analysis of SAXS frames for the three AB-GAPDH samples: inactive (light grey symbols, maximum at $13 \mathrm{ml}$ ), active-short (grey symbols, maximum at $14.8 \mathrm{ml}$ ) and active (black symbols, maximum at $15.4 \mathrm{ml}$ ), are shown as a function of the SEC elution volume. The datapoints belonging to the frames averaged to obtain the selected scattering profiles are highlighted with a colour code. The elution profile given by the scattering intensity at zero angle $(\mathrm{I}(0)$, circles, right ordinate axis) is plotted together with the radius of gyration $\left(R_{g}\right)$ obtained from the Guinier approximation (diamonds, left ordinate axis). In the inset, molecular weight ( $\mathrm{MW}$ ) estimated from the Porod volume ( $\mathrm{MW}\left(\mathrm{V}_{\mathrm{P}}\right)$, diamonds). The MWs expected on the basis of the protein sequence for $\left(A_{2} B_{2}\right)_{n}$ oligomers with $n=1,2,4$ and 5 are reported as dashed lines for reference. $P(r)$ functions calculated from the selected scattering profiles in the elution of the samples: (B) inactive, (C) active-short, (D) active; in the insets the elution profiles given by the SAXS integrated intensity are also shown. 


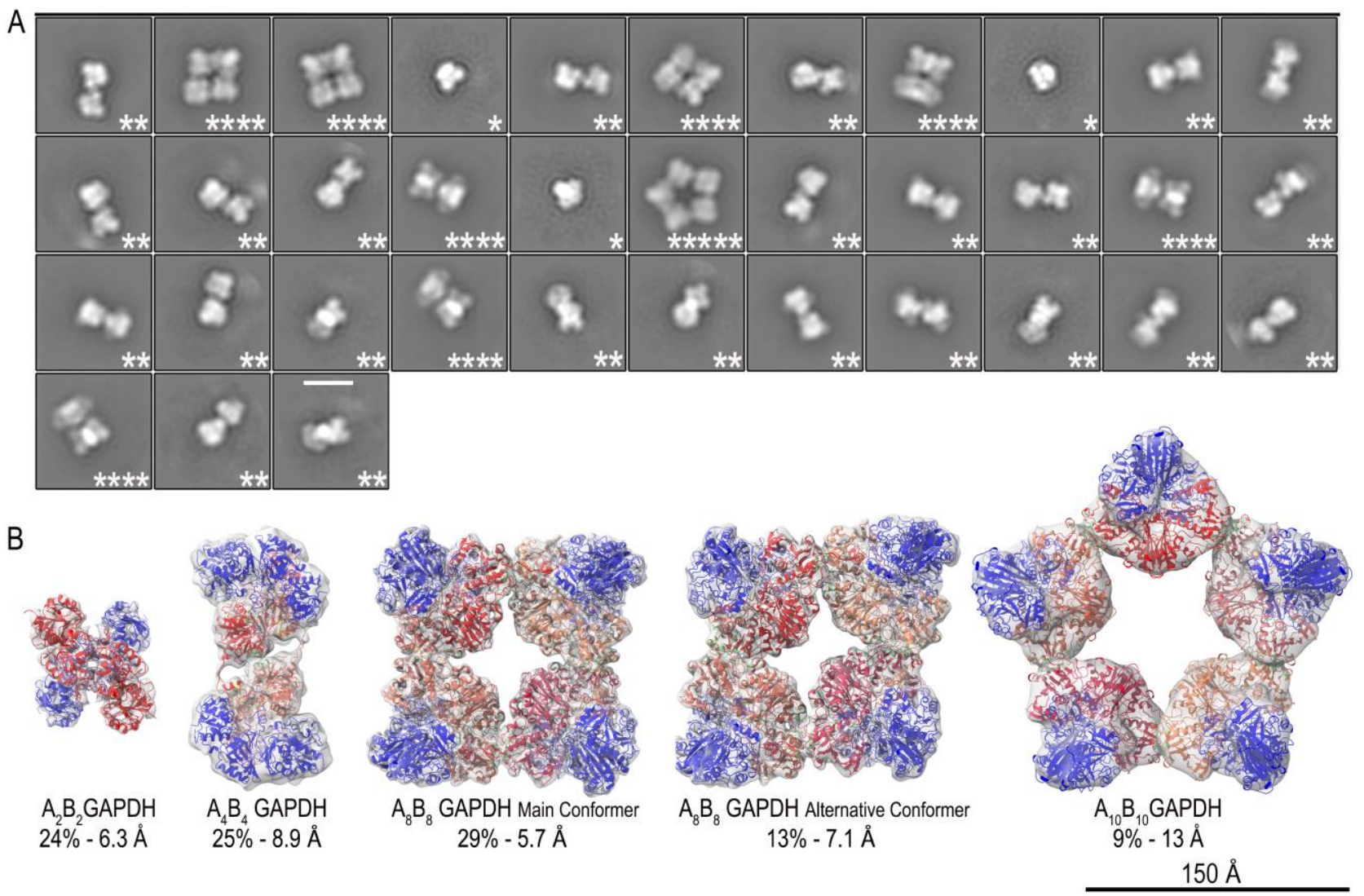

Figure 2. AB-GAPDH oligomers.

(A) Representative single-particle 2D classification obtained from the complete GAPDH data set showing the presence of class averages attributable to $A_{2} B_{2}, A_{4} B_{4}, A_{8} B_{8}$ and $A_{10} B_{10}$ oligomers. For each species, the number of $A_{2} B_{2}$ tetramers is indicated by asterisks. The scale bar is $150 \AA$. (B) GAPDH oligomer cryoEM density maps fitted with models derived from the crystal structure of the oxidized $A_{2} B_{2}$ complexed with $\mathrm{NADP}^{+}$(PDB ID code 2PKQ) (21). The O/Q, A/C, E/G, K/I and M/S B-subunits are represented in red, tomato, crimson, coral and indian red, respectively. The A-subunits are in blue. The numbers below the cryoEM electron density maps represent the oligomer relative abundances and their resolutions, respectively. 

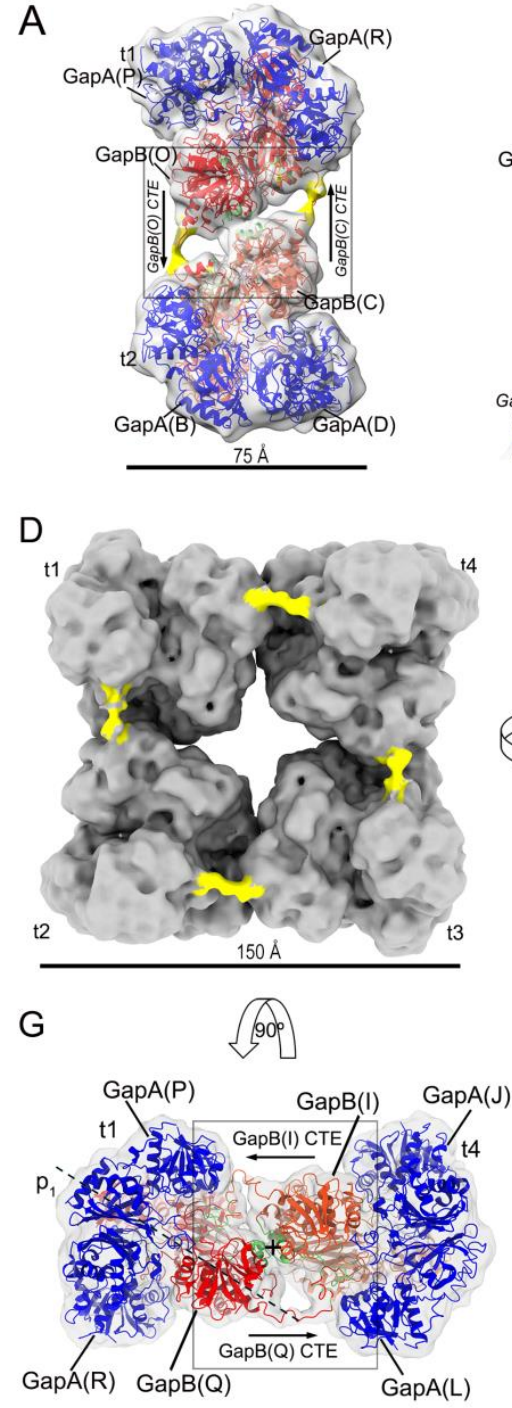
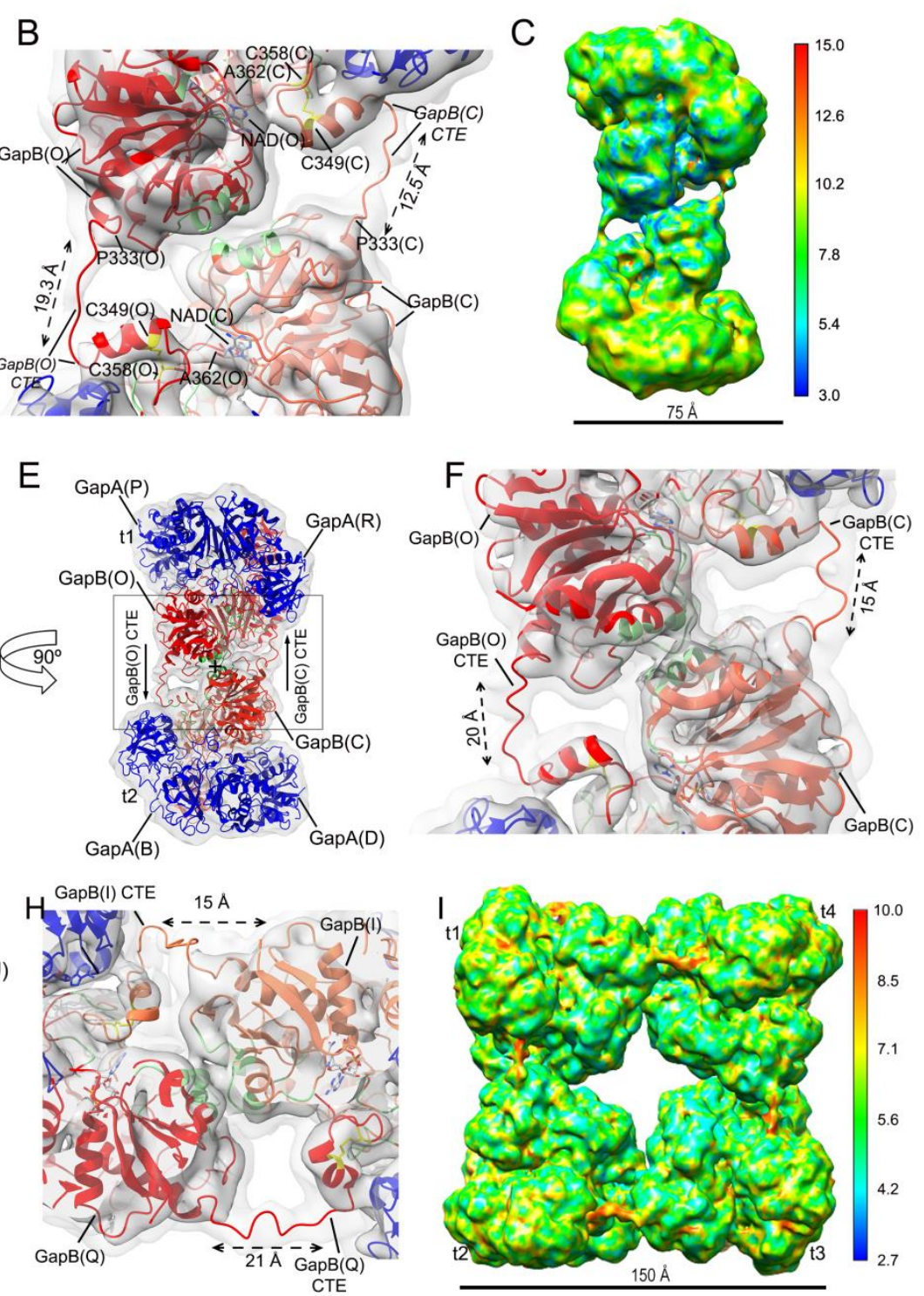

Figure 3. $A_{4} B_{4}$ and $A_{8} B_{8}$ oligomers.

(A) CryoEM density map of the $\mathrm{A}_{4} \mathrm{~B}_{4}$ oligomer at $8.9 \AA$ resolution. The map, shown at low density threshold, reveals two regions (highlighted in yellow) connecting the $t 1$ and $t 2 A_{2} B_{2}$ tetramers. (B) Detail of the region boxed in (A). (C) CryoEM electron density map of the $A_{4} B_{4}$ oligomer filtered according to ResMap local resolution. (D) CryoEM electron density map of the $\mathrm{A}_{8} \mathrm{~B}_{8}$ oligomer shown at a low density threshold. Note the connecting regions (highlighted in yellow) among the GAPDH tetramers t1-t4. (E) Side view of the maps in (D) showing the $t 1$ and $t 2$ tetramers. $(F)$ Detail of the boxed region in (E). (G) Side view of the map in (D) showing the $\mathrm{t} 1$ and $\mathrm{t} 4$ tetramers. $(\mathrm{H})$ Detail of the region boxed in $(\mathrm{G})$. (I) CryoEM electron density map of the $\mathrm{A}_{8} \mathrm{~B}_{8}$ oligomer filtered according to ResMap local resolution. All maps are fitted with their corresponding model derived from the crystal structure of the oxidized $\mathrm{A}_{2} \mathrm{~B}_{2}$ complexed with $\mathrm{NADP}^{+}$(PDB ID code 2PKQ) (18). The $O / Q, A / C, E / G, K / I$ and $M / S$ B-subunits are represented in red, tomato, crimson, coral and indian red, respectively. The A-subunits are in blue. In $(B),(F)$ and $(H)$, the densities of the $3 D$ reconstructions are displayed at two different isosurface levels (higher in dark gray and lower in light gray) and the interfacing residues between adjacent GAPDH tetramers are highlighted in green. 

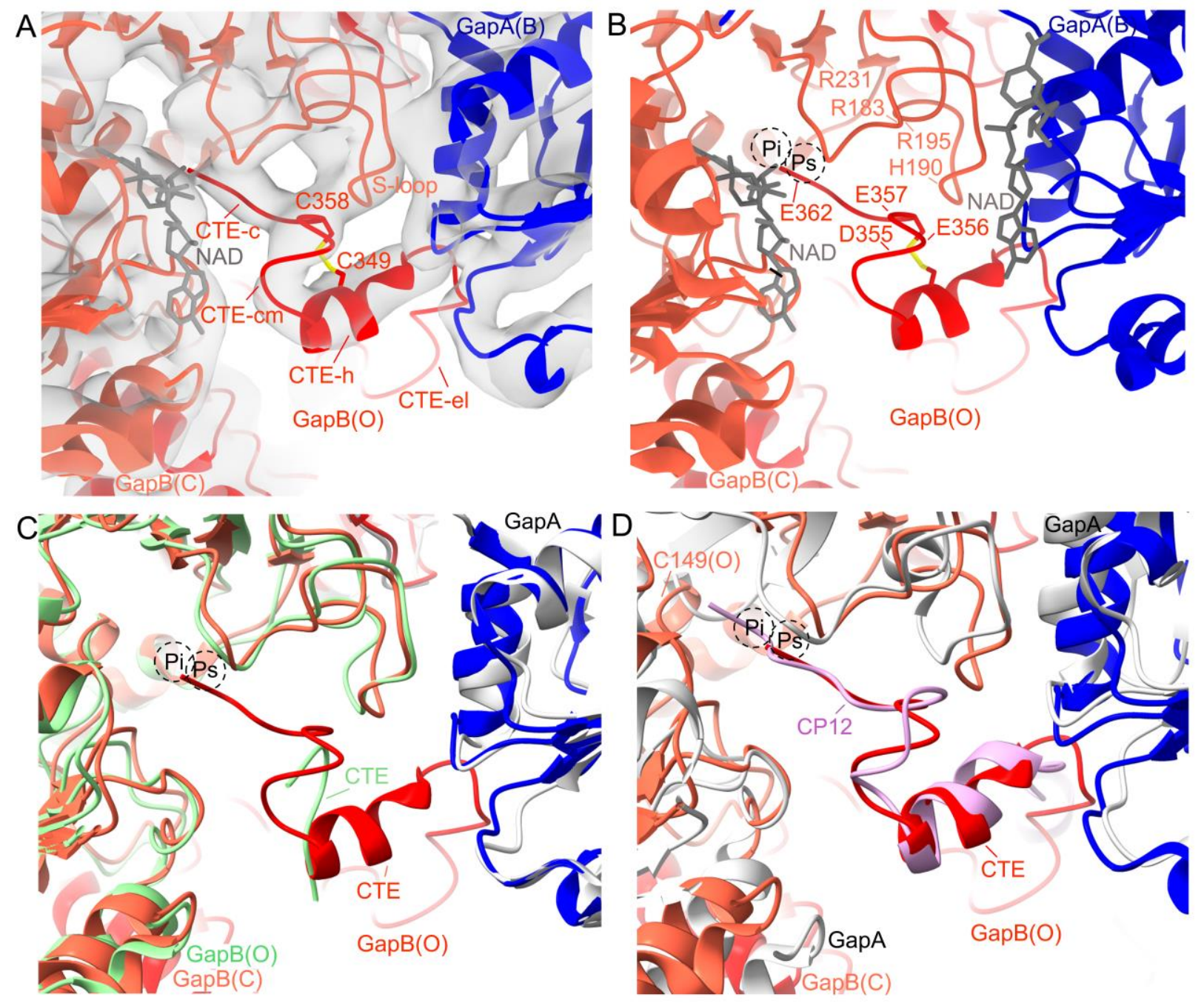

Figure 4. The CTE in the $A_{8} B_{8}$ oligomer.

(A) Detail of the CTE of B-subunit (chain $O$ ) in red inserted in the active site of B-subunit (chain $C$ ) in tomato, of the adjacent $\mathrm{A}_{2} \mathrm{~B}_{2}$ tetramer. The A-subunit (chain B) is in blue. CTE-el: CTE extended linker; CTE-h: CTE helix; CTE-cm: CTE circular motif; CTE-c: CTE random coil. (B) Detail of the CTE of B-subunit (chain O) in red inserted in the active site of $B$-subunit (chain $C$ ) in tomato, of the adjacent $A_{2} B_{2}$ tetramer. The A-subunit (chain $B$ ) is in blue. The negatively charged residues of CTE likely interacting with the positively charged residues of $\mathrm{B}$-subunit are indicated. The NAD+ bound to the A-subunit is also shown. The $\mathrm{P}_{\mathrm{s}}$ and $\mathrm{P}_{\mathrm{i}}$ labels indicate the substrate binding site. $(C)$ Detail of the CTE of $B$-subunit (chain $O$ ) in red superimposed to the CTE of $B$-subunit (chain $O$ ) in green from the crystal structure of the oxidized $\mathrm{A}_{2} \mathrm{~B}_{2}$ complexed with NADP+ (PDB ID code 2PKQ) (21). The $B$-subunit (chain $O$ ) and the A-subunit of $A_{2} B_{2}$ crystal structure are in green and light grey, respectively. Colour code for cryoEM structure is as in panels $(A)$ and $(B)$ Note that the two CTEs shows a different conformation and the CTE from $\mathrm{A}_{2} \mathrm{~B}_{2}$ crystal structure ends in the more external region of the catalytic cavity, far away the substrate binding site ( $P_{s}$ and $P_{i}$ sites). (D) Detail of the CTE of $B$ subunit (chain $\mathrm{O}$ ) in red superimposed to the $\mathrm{CP} 12 \mathrm{C}$-terminal domain in violet, from the cryoEM model of the ternary GAPDH-CP12-PRK complex (PDB ID 6GVE) (27). The A-subunits of GAPDH from the complex crystal structure are shown in light grey. The catalytic Cys149 is indicated. Note that CTE and the C-ter domain of CP12 have a very similar conformation and CP12 fills both the $\mathrm{P}_{\mathrm{s}}$ and the $\mathrm{P}_{\mathrm{i}}$ sites differently from CTE which ends in the Ps. 